\title{
Pediatric brain repair from endogenous neural stem cells of the subventricular zone
}

\author{
Yusuke Niimi ${ }^{1}$ and Steven W. Levison ${ }^{1}$
}

There is great interest in the regenerative potential of the neural stem cells and progenitors that populate the germinal zones of the immature brain. Studies using animal models of pediatric brain injuries have provided a clearer understanding of the responses of these progenitors to injury. In this review, we have compared and contrasted the responses of the endogenous neural stem cells and progenitors of the subventricular zone in animal models of neonatal cerebral hypoxia-ischemia, neonatal stroke, congenital cardiac disease, and pediatric traumatic brain injury. We have reviewed the dynamic shifts that occur within this germinal zone with injury as well as changes in known signaling molecules that affect these progenitors. Importantly, we have summarized data on the extent to which cell replacement occurs in response to each of these injuries, opportunities available, and obstacles that will need to be overcome to improve neurological outcomes in survivors.

$\mathbf{P}$ ediatric brain damage often leads to significant morbidity and severe long-term neurologic and cognitive deficits, such as cerebral palsy, epilepsy, behavioral disorders, impaired vision, and language function. More than half of all children with cerebral palsy are born at term and in many instances the etiology is related to some form of cerebrovascular focal or global insult (1). There is great interest in the regenerative potential of the neural stem cells and progenitors that populate the germinal zones of the immature brain. Studies using animal models of pediatric brain injuries have provided a clearer understanding of the responses of these progenitors to injury. As the literature on this subject is quite large, in this review we will compare and contrast the responses of the endogenous neural stem cells and progenitors that reside in the subventricular zone (SVZ) to neonatal hypoxic-ischemic injury, neonatal stroke, neonatal cardiac insufficiency, and pediatric traumatic brain injury.

THE STEM CELLS AND PROGENITORS OF THE PEDIATRIC SVZ The cells that comprise the SVZ are mix of the primitive neuroepithelial cells that populated the embryonic ventricular zone and a variety of intermediate progenitors. The radial neuroepithelial progenitors (NEPs), which undergo interkinetic movement, decrease in abundance as the brain develops, while the disorganized, unipolar progenitors of the SVZ accumulate rapidly as a result of extensive cell proliferation. Indeed, $90 \%$ of mouse SVZ cells are actively dividing at $\sim 2 / 3$ of gestation in the mouse (2). The SVZ peaks in size during the first week of postnatal development in rodents and at about the 35th week of gestation in humans (3-7). Early SVZ cells are predominantly neurogenic during fetal development, and preferentially produce GABAergic interneurons in the forebrain as well as GABAergic and dopaminergic interneurons of the olfactory bulb (8). There appear to be six streams of migrating cells emanating from the SVZ during discrete periods of postnatal neurodevelopment. These are (i) the rostral migratory stream to populate the olfactory bulb with interneurons (9); (ii) the medial migratory stream that populates the prefrontal cortex (10); (iii) the ventral migratory mass that migrates along the ventrocaudal migratory stream and populates the Islands of Calleja in the nucleus accumbens (11); (iv) another ventral migratory stream that populates the claustrum (12); (v) the dorsal migratory pathway to the hippocampus directed towards the occipital cortex (13); and (vi) migration from the most dorsal region of the SVZ to produce upper layer glutamatergic neurons (14).

Fate mapping experiments have established that the NEPs are heterogeneously distributed along the dorsoventral borders of the ventricle, with different types of interneurons being produced from geographically restricted regions (15-17). Gene expression analyses have further defined differences in the progenitors residing in the dorsal vs. the ventrolateral regions of the SVZ (18). While the SVZ maintains its capacity to produce neurons across the lifespan, in late gestation and in the early postnatal period, SVZ cells are predominantly gliogenic, first generating astrocytes and later oligodendrocytes. These glial cells are produced from bipotential neuron/glial progenitors $(19,20)$, as well as from restricted progenitors that are destined to become only glial cells $(8,21)$. Once gliogenesis begins to slow down, the SVZ begins to involute (3).

The organization of the adult SVZ has been an area of intense study. Early studies used light and electron

\footnotetext{
'Laboratory for Regenerative Neurobiology, Department of Pharmacology, Physiology and Neuroscience, Rutgers-New Jersey Medical School, Newark, New Jersey. Correspondence: Steven W. Levison (levisosw@rutgers.edu)

Received 21 July 2017; accepted 5 October 2017; advance online publication 8 November 2017. doi:10.1038/pr.2017.261
} 
microscopy to parse the rodent SVZ into three cell types that were thought to be either astrocytes or oligodendrocyte progenitors $(22,23)$. With the discovery that there are slowly dividing cells in the adult SVZ that possess the properties of stem cells and that these stem cells are regularly producing new olfactory bulb neurons, much attention has focused on the cells that comprise the adult SVZ. In a seminal article published in 1997, Doetsch et al. (24) used ultrastructure and immunohistochemistry to divide the cell types of the adult SVZ into six cell types (excluding resident microglia) and the terminology that they introduced has been widely adopted. They assigned an alphabetic nomenclature to define the cells within the SVZ. The NSCs were termed type $\mathrm{B}_{1}$ cells; immature astrocytes, type $B_{2}$; transit amplifying cells, type $C$; neuroblasts, type A; tanycytes, type $\mathrm{D}$; and ependymal cells, type E cells (24).

While the nomenclature introduced by Doetsch et al. (24) has been widely adopted, it is inadequate for describing the cells residing in the pediatric SVZ. Missing from that classification scheme are glial-restricted progenitors as well as oligodendrocyte progenitors, which are prominent in the neonatal SVZ $(19,20)$. Our lab established a different classification scheme for the NSCs and progenitors of the pediatric SVZ that was derived from flow cytometric and clonal analyses of neonatal, adolescent, and adult SVZ cells (Table 1) (25). Using four surface antigens, we parsed the SVZ into eight different types of multipotential and bipotential progenitors. However, this panel excluded cells that were already restricted to astrocytes, neurons, ependymal cells, and oligodendrocyte progenitors. Accordingly, the neonatal SVZ has to be comprised of at least 14 different types of progenitors. Indeed, more recently, we added three additional cell surface markers to the panel (A2B5, O4, GLAST), enabling us to parse the cells of the neonatal SVZ into more than 75 different subtypes (26). The extent to which each of these 75 antigenically defined cells represents a unique progenitor vs. similar progenitors at different developmental stages remains to be determined. Single-cell gene profiling studies on sorted and unsorted neonatal SVZ cells are in progress, which will enable us to refine our current classification schemes toward defining the intermediate progenitors involved in neural development.

\section{Species Differences in the SVZ}

While a significant portion of the work on neural stem cells and progenitors of the SVZ has been conducted in rodents the SVZ of gyrencephalic mammals has a different structure. In all gyrencephalic species studied to date, which include rabbits, ferrets, pigs, monkeys, and humans, the neonatal periventricular region can be divided into four compartments: (1) the ependymal layer that lines the ventricles; (2) the inner SVZ that is comprised of densely packed neuroblasts and immature glial cells; (3) an astroglial fiber layer; and (4) the outer SVZ, which contains chains of doublecortin-positive $(\mathrm{Dcx}+)$ cells $(27-31)$. The SVZ is mostly devoid of mature neurons, and as an important germinal zone, it contains cells that stain for Ki-67, which is a marker of cell proliferation. Indeed, even dolphins, which do not have an olfactory bulb, have a vestigial SVZ that maintains this inner and outer SVZ organization (32). It seems that only rodents (both rats and mice) have an inner ventricular zone and lack the outer SVZ. More recent studies on the fetal primate SVZ have shown that this significantly expanded outer SVZ is comprised of outer radial NEP cells and additional intermediate progenitors. These outer radial NEP cells are a transient population that produces a large number of upper layer pyramidal cells characteristic of the brains of more evolved mammals (33).

Another difference between the rodent brain and the human brain is that it was once postulated that the adult human SVZ produces olfactory bulb interneurons throughout life; however, several recent studies have failed to provide support for this conclusion. Rather, it appears that the production of olfactory bulb neurons from SVZ progenitors becomes undetectable after $\sim 2$ years of age (10). Carbon dating studies by Bergman et al. (34) set a limit of $<1 \%$ of the neurons in the human olfactory bulb being exchanged over 100 years (34). Therefore, while the adult SVZ has the potential to make new neurons (35), its primary function is to serve as a source for new glial cells (36). An unexpected and unconfirmed finding from the carbon-dating studies is that

Table 1. Neural progenitors of the neonatal mouse SVZ

\begin{tabular}{|c|c|c|c|}
\hline Antigenic profile & Developmental potential & Growth factor responsiveness & Designation \\
\hline CD133+LeX+NG2-CD140a- & Multipotential & EGF and FGF-2 & NSC \\
\hline CD133-LeX+NG2-CD140a- & Multipotential & EGF and FGF-2 & MP1 \\
\hline CD133+LeX+NG2+CD140a- & Multipotential & EGF and FGF-2 & MP2 \\
\hline CD133-LeX+NG2+CD140a- & Bipotential & EGF and FGF-2 & BNAP/GRP1 \\
\hline CD133+LeX+NG2+CD140a+ & Multipotential & PDGF+FGF- $2+0.5 \%$ FBS & MP4 \\
\hline CD133-LeX-NG2+CD140a- & Multipotential/bipotential & PDGF+FGF-2 & MP3/GRP2 \\
\hline \multicolumn{4}{|c|}{$\begin{array}{l}\text { BNAP, bipotential neuron and astrocyte progenitor; FGF-2, fibroblast growth factor-2; GRP, glial-restricted progenitor; MP, multipotential progenitor; NSC, neural stem cell; PDGF } \\
\text { platelet-derived growth factor; SVZ, subventricular zone. } \\
\text { The stem cells and progenitors of the neonatal SVZ were distinguished on the basis of their cell surface antigen, growth factor responsiveness, and differentiation potential as as } \\
\text { originally described by Buono et al. (25). Cells types are: NSCs; MPs; BNAPs; bi-potential GRPs. Cells characterized as multipotential produced neurons, astrocytes, and oligoden- } \\
\text { drocytes. The GRPs produced both astrocytes and oligodendrocytes (GRP1, GRP2, and GRP3). }\end{array}$} \\
\hline
\end{tabular}


new neurons are produced in the adult human basal ganglia (presumably from the SVZ) (37).

\section{INJURIES CAUSING BRAIN DAMAGE IN CHILDREN AND RELEVANT ANIMAL MODELS \\ Hypoxic-Ischemic Encephalopathy}

Hypoxic-ischemic encephalopathy (HIE) arises from inadequate oxygenation/perfusion to the fetus or from asphyxiating complications during birth with a prevalence rate ranging from 2 to 4/1,000 live births (38). This injury represents a major cause of neurological morbidity in infants, producing cognitive, motor, and sensory deficits that vary depending upon maturation state at the time of onset of the HIE and the severity of the brain damage. Presently, there is only one therapeutic provided to pregnant women to try to prevent HIE and the benefits of this therapeutic are dubious (39). Therapeutic hypothermia is an approved treatment for moderate neonatal encephalopathy and has been shown to improve survival if administered within the first $6 \mathrm{~h}$ of life $(40,41)$. However, the therapy offers limited functional recovery and comes with its own set of complications. As there is no approved therapy for preterm infants, too often they face lifelong learning disabilities and impairment $(42,43)$. Consequently, therapies are desired that work synergistically with hypothermia or that are effective when used alone to restore the normal course of brain development.

Responses of SVZ cells in animal models of HIE: Given the restorative potential of stem cells to repair damaged tissues throughout the body, neurobiologists have been asking how the cells of the SVZ are affected by HIE and whether the resident neural stem cells and progenitors of the SVZ can replace those cells that are damaged. These studies have established that during the first $24-48 \mathrm{~h}$ after moderate to severe injury, there is extensive cell death within the SVZ (44). With additional analyses, it was found that during these first 2 days of recovery from $\mathrm{H}-\mathrm{I}$, progenitors, and especially oligodendrocyte progenitors within the SVZ die excitotoxic and apoptotic cell deaths. Using immunohistochemical markers for stem cells and progenitors combined with positional information, those cells in the most medial region of the SVZ, which expressed Nestin, but do not express PSANCAM (stem cells), survived severe perinatal H-I insults, whereas cells expressing PSA-NCAM and negative for Nestin (neuroblasts and bi-potential glial progenitors), as well as some oligodendrocyte progenitors were vulnerable (45). A similar phenomenon was also observed in the mouse brain where more severe insults lead to decreased cell proliferation in the SVZ (46).

At later intervals of recovery, the size of the SVZ increases (47-49). Interestingly, this increase in the area of the SVZ is proportional to the extent of hemispheric damage and is a result of increased cell proliferation $(48,50)$. These new progenitors form tripotential, self-renewing neurospheres in vitro, indicating that the increase is relatively specific for the most undifferentiated progenitors. In their 2006 paper, Felling et al. (50) provided evidence implicating Notch signaling in the expansion of the neural progenitor (NP) pool. More recently, Felling et al. (51) used an in vitro model for $\mathrm{H}-\mathrm{I}$, hypoglycemia, and hypoxia $(\mathrm{H}-\mathrm{H})$, and showed that $\mathrm{H}-\mathrm{H}$ itself is not sufficient to induce Notch-1, but that $\mathrm{H}-\mathrm{H}$ induces astrocytes to produce leukemia inhibitory factor, which in turn increases Notch1 expression in the neural stem/ progenitors (NSPs). There is a controversy as to whether neonatal H-I increases the proportion of bona fide neural stem cells vs. progenitors. Studies by a number of laboratories have revealed increases in neural progenitors after recovery from neonatal $\mathrm{H}-\mathrm{I}$. To better understand which NPs expanded after neonatal $\mathrm{H}-\mathrm{I}$, Buono et al. $(26,52)$ used a four-color multimarker flow cytometry panel to quantify the relative proportions of eight unique NPs after injury in the neonatal mouse. They discovered that the NSCs were significantly diminished as a consequence of $\mathrm{H}-\mathrm{I}$, whereas the number of MP2 multipotential progenitors increased as did the proportions of three types of glial-restricted progenitors at $48 \mathrm{~h}$ after injury (52). Interestingly, some of the progenitor types that increased after $\mathrm{H}-\mathrm{I}$ were epidermal growth factor receptor+ (EGFR+) - cells, which is consistent with a previous report that showed that that NG2+/EGFR+ cells increased in abundance after $\mathrm{H}-\mathrm{I}$ in the neonatal rat (53).

\section{Prospects for repair and cell replacement after perinatal $\mathrm{H}-\mathrm{I}$ :} While it is clear that new brain cells are being produced in response to these injuries, data compiled to date suggest that they are not capable of fully replacing the cohort of neurons that were damaged. Early studies used bromodeoxyuridine (BrdU) to label cells that had divided after injury in combination with markers for immature and mature neurons to demonstrate that new neurons were being produced. Using Dcx, an immature neuronal marker, combined with BrdU to label cells that had recently proliferated, Dcx/BrdU double positive were observed streaming from the immature mouse and rat SVZ into the adjacent damaged striatum at 1 week following neonatal $\mathrm{H}-\mathrm{I}(47,48)$. At 14 days of recovery some BrdU+ cells expressed NeuN (a mature neuron marker). Disappointingly, by 3 weeks of recovery in the mouse model of $\mathrm{H}-\mathrm{I}$, no BrdU+ cells expressing the neuronal markers MAP2, TuJ1, or NeuN could be found, indicating that the vast majority of the new neurons that were generated failed to survive. Supporting this interpretation, Plane et al. (2004) identified newly generated neurons that were dying apoptotic deaths at 2 weeks of recovery. ${ }^{48}$ These findings suggest that whereas neurogenesis is initiated in the newborn mouse brain that the long-term survival of these newly generated neurons is not supported.

By contrast, in the neonatal rat brain, not only are new neurons are being produced after $\mathrm{H}-\mathrm{I}$ but these new neurons seed both the injured striatum and the injured neocortex (54). Yet, another difference between mice and rats is that in the rat 


\section{Review | Nimi and Levison}

brain these new neurons are continuously produced for at least for 2 months after the injury, as BrdU injections into animals at 2 months of recovery labeled cells that subsequently differentiated into NeuN+ neurons located in the neocortex within the penumbra of the injury (54). However, as encouraging as these results were, subsequent analyses of the types of neurons that were produced indicated that these new neurons were almost exclusively interneurons, and more specifically, that they were the subset of neurons that expressed calretinin. Indeed, even the neuroblasts that migrated into the striatum became calretinin+ interneurons. They did not express DARPP-32, calbindin-D-28K, parvalbumin, somatostatin, or choline acetyltransferase (55). Importantly, using retroviral-mediated gene transfer to fate map SVZ cells, Yang et al. (54) established that these new neurons are derived from the SVZ (54). There are little data to support the conclusion that they arise from dividing progenitors residing outside of the SVZ. Similar to the results obtained in mouse models of $\mathrm{H}-\mathrm{I}$, very few $(\sim 15 \%)$ of the newly generated neurons are retained, but of those retained, some persist for at least 5 months (56).

Whereas there has been a surge of studies examining neuronal replacement after perinatal $\mathrm{H}-\mathrm{I}$, there have been fewer studies assessing the extent of oligodendroglial replacement. Zaidi et al. (57) used BrdU labeling at 21 days of recovery from perinatal $\mathrm{H}-\mathrm{I}$ in the rat and documented an increase in the number of newly generated oligodendrocytes in the damaged hemisphere and suggested that these new oligodendrocytes are produced by SVZ cells. Ong et al. (47) observed an increase in oligodendroglial proliferation 4 weeks after H-I. Yang et al. (54) found that of the newly produced cells migrating out of the SVZ after $\mathrm{H}-\mathrm{I}$ that $\sim 1 / 3$ differentiated into neurons, $1 / 3$ into astrocytes, and $1 / 3$ became oligodendrocytes. However, none of these studies established whether these newly produced oligodendrocytes proceeded to differentiate into myelin-producing cells, which remains an important issue for study.

As new oligodendrocytes are produced, the failure of myelin production must be due to the inability of these immature oligodendrocytes to differentiate, their transdifferentiation into astrocytes or neurons, or axonal loss. There is clearly axonal loss in the subcortical white matter after perinatal H-I; however, there are data showing that the paucity of oligodendrocytes is disproportional to the degree of axonal loss (44), suggesting that their failure to differentiate is not due to the absence of requisite axons. There is significant astrogliosis after perinatal $\mathrm{H}-\mathrm{I}$ along with an increase in BrdU/glial fibrillary acidic protein (GFAP)+ cells $(44,57,58)$. These results point to the proliferation of astrocytes and it is well established that reactive astrocytes produce extracellular matrix components such as hyaluronic acid and chondroitin sulfate proteoglycans that inhibit oligodendrocyte differentiation $(59,60)$. There are also numerous cytokines that appear to be produced by astrocytes that inhibit oligodendrocyte progenitor cell maturation that include fibroblast growth factors, WNTs, endothelin-1, and Notch ligands (61-64). An alternative explanation for the lack of myelination is problems in SVZ cell specification. Bain et al. (65) investigated gliogenesis using replication deficient retroviruses and found that astrocyte generation was disproportional to oligodendrocyte production after neonatal H-I. Moreover, they found that elevated levels of transforming growth factor- $\beta$ (TGF- $\beta$ ) were produced in the $\mathrm{H}-\mathrm{I}$ brain and that the aberrant levels of GFAP in the SVZ could be reduced by administering an antagonist to the TGF- $\beta$ receptor, activin-like kinase receptor5 (65). In a recent study by Sabo et al. (66), they found evidence for increased bone morphogenetic protein signaling in Olig1+ SVZ cells, which correlated with decreased production of oligodendrocytes and increased production of interneurons (66).

\section{Pediatric Traumatic Brain Injury}

Traumatic brain injury (TBI) is one of the most common causes of acquired disability during childhood, with epidemiological reports suggesting that 750 out of 100,000 children will suffer a TBI each year. While less than half will seek medical care, $10 \%$ will be hospitalized and $7 \%$ will sustain significant head injury (67). TBI refers to a mechanically induced physiologic disruption of brain function. That physiologic disruption may occur as a result of cerebral contusions following the impact of the brain with the internal surface of the skull, accompanied by shear-force injuries within the cerebral white matter as a result of rapid rotational acceleration and deceleration. With advances in medical treatment, mortality rates are low, and decreasing, and there are ever-increasing numbers of survivors. However, the financial burden is enormous, estimated at $\$ 1$ billion USD/ year in hospital costs (68). Children who sustain severe TBI are clinically managed to prevent swelling and further damage from swelling. Resources are limited after the acute recovery stage, despite good evidence that childhood TBI has more serious and persisting effects than similar adult insults. This increased vulnerability in childhood is thought to be due to the decreased threshold for apoptosis in the immature brain and the potential of early insults to derail ongoing brain development and to disrupt cognitive development $(69,70)$. Mechanical injuries cause immediate cellular damage as well as damage to axons and blood vessels, which cause either focal or diffuse patterns of cell loss. Secondary effects, that can include hypoxia, ischemia, edema, and increased intracranial pressure, can significantly amplify the extent of damage (70).

Differences in the magnitude of the SVZ progenitor proliferative responses have been observed when comparing injury models. In an aspiration TBI model, there were significantly greater increases in BrdU+ SVZ cells in adults than seen in the controlled cortical impact (CCI) model (71). One explanation for these differences might be that aspiration TBI produces more widespread damage that affects a greater percentage of the injured hemisphere than focal CCI produces. This suggests that injury signals are produced in the damaged hemisphere and that the larger the injury the greater the stimulus. Species differences in responses to TBI 


\section{SVZ cell responses to brain injuries | ReView}

are also apparent. For example, a greater proliferative response is seen in mice compared to rats with the CCI model of TBI (72).

Responses of SVZ cells and prospects for repair in animal models of pediatric TBI: Changes in SVZ cell proliferation have been examined across a number of animal models of traumatic brain injury, including penetrating brain injuries, aspiration lesions, fluid percussion injury, and CCI, but until recently, most of those studies have been performed on adult animals. Goodus et al. (72) used a variety of approaches to unmask the identity of the cells that were proliferating in the SVZ after neonatal and adolescent traumatic brain injuries induced by a CCI (72). Using immunofluorescence for Nestin, Ascl-1 (multipotential progenitors marker), and Ki67 (proliferating cell marker), they found that by $48 \mathrm{~h}$ after the injury there were increased numbers of Nestin+/Ascl-1-/Ki-67+ (NSC) cells residing immediately adjacent to the ependymal cells in the medial aspect of dorsolateral SVZ after TBI. In contrast, there was less proliferation of the Nestin+/Ascl-1+ (multipotential progenitors) cells residing throughout the SVZ. Using the four-marker flow cytometry panel described earlier, Goodus et al. (72) quantified the relative percentages of NSCs and progenitors residing in the SVZ after TBI in the adolescent mouse (72) and found that CCI doubled the number of both bona fide NSCs, decreased the proliferation of one subclass of multipotential progenitor, and increased the proportions of two other subclasses of multipotential progenitors. Interestingly, Goodus et al. (72) saw a different set of progenitors responding to CCI vs. H-I. Notably, they saw increases in the proportion of NSCs after CCI injury, whereas, as discussed earlier, the NSCs become less abundant after perinatal H-I $(25,52)$. Furthermore, there was a significant expansion of glial progenitors after $\mathrm{H}-\mathrm{I}$, whereas the glial progenitors were not as significantly amplified after CCI. However, one similarity was the increase in the progenitors classified as MP3/GRP2 cells. Interestingly, these are EGF-responsive progenitors, suggesting that increases in either EGF ligands or the EGFR could be responsible for the amplification of these progenitors. Supporting this hypothesis, ligands for the EGFR, such as heparin-binding EGF-like growth factor, which is expressed at high levels in the brain, as well as TGF- $\alpha$ increase after cerebral injury (73).

Costine et al. (28) used a model of blunt impact head injury to the rostral gyrus of piglets to evaluate the responses of the SVZ cells. Reminiscent of studies of $\mathrm{H}-\mathrm{I}$ in rodents, they observed an increase in the area of the SVZ in piglets developmentally similar to infants (postnatal day 7) and toddlers (postnatal day 30), but no change in the size of the SVZ in piglets developmentally similar to human preadolescents (4 months of age) (28). In a subsequent study, Taylor et al. (2013) evaluated the total numbers of Dcx+ cells and newly generated Dcx+ neuroblasts within the subcortical white matter found between the SVZ and the rostral gyrus, as well as within the gray matter of the rostral gyrus (12). They found that there was no increase in the number of neuroblasts within the white matter near the SVZ at 7 days after the injury, but that the neocortical impact increased the density of neuroblasts in the injured rostral gyral gray matter with a trend toward an increase in neuroblasts in the rostral gyral white matter in those animals with cavitating lesions. Using BrdU labeling they found that the majority of these neuroblasts had been produced before the injury rather than being produced after the injury. However, a limitation of their study was that they injected BrdU for only 2 days after the injury, so they might have missed delayed responses from the progenitors of the SVZ. Within the white matter, the neuroblasts aligned themselves along the edge of the lesion cavity and some of the Dcx cells were multipolar, suggesting that they were differentiating. Whereas there were large numbers of Dcx + cells within the white matter, the neuroblasts produced after the injury represented $<1 \%$ of these cells.

Despite the increased capacity for SVZ cells in young and adolescent-aged animals to proliferate and to produce more NSCs and multipotential progenitors, studies have shown that these responses ultimately fail to produce large numbers of new neurons in the injured neocortex. For example, Goodus et al. (72) observed many BrdU+/Dcx+ cells that appeared to be migrating out of the SVZ into the neocortex 2 weeks after CCI in postnatal day 11 rats. However, virtually no BrdU+ mature neurons were seen in the gray matter adjacent to the lesion in the cohort of animals that were allowed to survive to 28 days after injury (72).

In a study of neurogenesis after TBI in humans, Taylor et al. (12) found that there was little evidence for the production of new neocortical neurons (based on Dcx staining). These results are clearly different from those in rodents where there is an increase in proliferating cells in the SVZ as well as numerous Dcx + cells migrating in the penumbra of the injury after TBI $(12,72,74)$.

\section{Perinatal Stroke}

Perinatal stroke is defined as a cerebrovascular event that occurs during fetal or neonatal life from 20 weeks gestational age to 28 days postnatally, with pathological or radiological evidence of focal arterial infarction of brain. In a population-based study with relatively frequent neuroimaging, unilateral neonatal stroke was recognized in 1-2 per 5,000 live infant births and the estimated mortality rate of neonatal stroke is 3.5/100,000 births annually. Although the majority of patients survive their stroke, $\sim 75 \%$ have sequelae including cerebral palsy, epilepsy, and a range of cognitive impairments $(75,76)$.

Neonatal stroke may present during the first 3 days of life with symptoms including focal motor seizures, apnea, and persistent feeding difficulties. Magnetic resonance imaging shows focal injury in arterial (70\%) or venous (30\%) distributions. Because recurrence rates are low, treatment of perinatal stroke in the acute setting has been largely supportive. The American Heart Association guidelines state that anticoagulation may be helpful in patients with perinatal 
stroke who have either a prothrombotic state or congenital heart disease (CHD) (77).

Responses of SVZ cells and prospects for repair in animal models of pediatric stroke: Studies using a neonatal stroke model induced by middle cerebral artery occlusion in P7 mice have observed apoptotic NPs within the SVZ during the first $24 \mathrm{~h}$ (78). However, in contrast to the studies described earlier on SVZ responses to neonatal $\mathrm{H}-\mathrm{I}$, in the pediatric model of stroke, the ischemic injury reduced both neurogenesis and gliogenesis. To study neurogenesis, P1 mice were injected with replication-incompetent lentiviruses intraventricularly to label the radial NEPs as well as the ependymal cells adjacent to the ventricles. The differentiation of these cells was then evaluated 2 weeks later. First, reduced numbers of olfactory bulb neurons were produced in the animals recovering from the stroke as well as reduced numbers of virally labeled astrocytes and oligodendrocytes in the striatum. Second, most of the new $\mathrm{Dcx}+$ neurons produced after this focal ischemic injury were not derived from the virally labeled radial NEPs, but were arising from more restricted NPs (78). As the authors of this study discuss, there may be significant differences in the injury parameters itself, such that a purely ischemic injury does not induce the same signals as an $\mathrm{H}-\mathrm{I}$ injury.

In a study that followed up on the work of Spadafora and colleagues, Gonzalez et al. (79) used the replication deficient virus to label the radial NEPs and then administered erythropoietin (Epo, 1,000 U/kg) upon reperfusion, $24 \mathrm{~h}$, and again 7 days later (79). They measured the density of NEP descended neurons, astrocytes, and oligodendrocytes in the striatum at $72 \mathrm{~h}$ and 2 weeks after stroke. They found that Epo treatment significantly increased the production of neurons and oligodendrocytes from the NEPs while reducing the number of astrocytes produced after middle cerebral artery occlusion. These data show that the responses of SVZ cells to pediatric stroke are clearly more subdued when compared with neonatal $\mathrm{H}-\mathrm{I}$; however, with appropriate activation by extrinsic signals such as Epo, there is increased SVZ cell proliferation, migration, and production of new neurons and astrocytes.

\section{Cardiac Insufficiency due to Congenital CHD}

Approximately 1 in every 150 infants born each year suffer from CHD (80). These cardiac anomalies reduce the flow of oxygenated blood to the brain with injury correlating with the severity of the malformation $(81,82)$. Although more than $75 \%$ of children with $\mathrm{CHD}$ who survive beyond the first year will live into adulthood, neurological deficits are common $(83,84)$.

Responses of SVZ cells in animal models of pediatric CHD and prospects for cell replacement: Since chronic cerebral hypoxia is common among CHD patients, the most commonly used mouse model of CHD used to evaluate the impact of CHD on the developing brain rears pups with their dams in an atmosphere of 9.5-10.5\% oxygen from P3 to P11.
This extended period of chronic postnatal hypoxia induces a general loss of neocortical and hippocampal volumes in the absence of detectable neuronal cell death. Analyses have established that this reduced brain volume can be attributed in part to smaller dendritic trees, reduced synaptogenesis, and decreased oligodendrocyte progenitor cell proliferation and maturation (86).

Using this rodent model for chronic hypoxia Fagel et al. (87) found that chronic hypoxia during postnatal days 3-11 results in a $30 \%$ loss of neocortical neurons that is reversed during recovery (87). In the first week of recovery, there is a marked increase in the number of new cells expressing immature astroglial markers in the SVZ. After 1 month of recovery, there are $40 \%$ more BrdU+ cells in the hypoxic neocortex with an observed ratio of differentiated cell types in both hypoxic and control animals of $45 \%$ oligodendrocytes, $35 \%$ astrocytes, and $10 \%$ neurons (87). Fate mapping studies performed using GFAP-Cre $\times$ GFP mice have shown that the radial NEPs of the SVZ and subgranular zone of mice maintained hypoxic from P3 to P11 continued to generate neurons for at least 2 weeks past when neurogenesis would have normally ceased. Moreover, these NEPs generated neocortical excitatory neurons that appeared to be incorporated into the synaptic circuits (88).

To investigate the causes of the abnormal white matter development, Jablonska et al. (89) investigated alterations in the intracellular signaling pathways within the oligodendrocyte progenitors. They found that the Cdk2 signaling pathway was more active in white matter oligodendrocyte progenitors during chronic hypoxia, while expression of negative regulator of cell proliferation, p27Kip1, and its regulator, FoxO1, were significantly reduced. Decreased p27Kip1 expression was also found in oligodendrocyte progenitors in the white matter of human newborns with neonatal hypoxic brain damage (89). In their more recent paper, Jablonska et al. (90) showed that with chronic hypoxia there is an increase in the expression and activity of the histone deacetylase Sirt1, resulting in deacetylation of the $\mathrm{Rb}$ protein that correlated with increased proliferation of the oligodendrocyte progenitors (90).

As white matter maturation delays are frequently seen in CHD patients (89), Scafidi et al. (91) sought to establish a therapeutic intervention that would help to restore white matter development. Following earlier studies that showed that EGF stimulated the proliferation of oligodendrocyte progenitors (92), they showed that intranasal administration of EGF from P11 to P14 after the chronic hypoxia prevented oligodendrocyte death, promoted oligodendrocyte generation, and improved behavioral recovery (91). Within a few weeks of recovery, the oligodendrocyte progenitors matured and myelination return to normal levels, but the myelin was abnormal, as detected by electron microscopy (89).

In parallel studies conducted in the neonatal piglet, Morton et al. (93) reported that chronic hypoxia from P3 to P11 (10.5\% oxygen chamber) impaired cortical development. For their studies the authors injected cell tracker green (CTG) into the SVZ to follow cell migration and differentiation, and 
stained the cells for Dcx and PSA-NCAM. In the chronic hypoxia condition, the number of CTG+ cells and CTG+/Dcx+ and $\mathrm{CTG}+/ \mathrm{PSA}-\mathrm{NCAM}+$ progenitors was significantly decreased. Hypoxia reduced proliferation within the SVZ, depleting a source of interneurons destined to migrate to the postnatal frontal cortex (93). These data suggest that in addition to deficits in white matter proliferation, chronic hypoxia also interferes with the migration of the progenitors from the SVZ; however, a caveat is that the $\mathrm{P} 3$ piglet brain is at a far more mature stage than a P3 mouse brain; therefore, it is difficult to compare and contrast the results from these studies.

\section{SIGNALING MOLECULES THAT ARE PRODUCED AFTER PERI-} NATAL BRAIN INJURY WITH KNOWN EFFECTS ON SVZ CELLS Multiple cytokines are produced as a result of perinatal brain injuries. Below, we will review those cytokines that are elevated after pediatric brain injuries and their actions on the neural progenitors of the SVZ. They are discussed in alphabetical order.

\section{Delta/Jagged/Notch-1}

Notch receptors are transmembrane proteins that are activated by membrane bound Delta and Jagged ligands and they are expressed by the stem cells and progenitors in the SVZ (94). Studies by Felling et al. $(50,51)$ showed that H-I increases the expression of Notch1 as well as one of its ligands, Delta-like 1, that results in increased expression of the downstream effectors, Hes1 and Hes5, within the NSPs of the SVZ. They further showed that pharmacologically decreasing Notch1 activity during the acute recovery period in vivo abrogated the increase in NSPs seen after neonatal H-I. Complementary studies have shown that intraventricular infusion of fibroblast growth factor-2 and Notch ligand deltalike 4 after stroke significantly increases the number of proliferating NSPs in the SVZ (95).

\section{Erythropoietin}

Epo is a $34-\mathrm{kDa}$ glycoprotein that binds to a homodimer of the Epo receptor (EpoR) present on the plasma membrane. In situ hybridization studies have shown that both Epo and the EpoR are present on the NEPs of the embryonic and fetal brain consistent with an important role in neurogenesis. Epo and EpoR germline null mice have smaller and less developed brains than their littermate controls, and in particular, the fetal SVZ of the developing forebrain is much reduced in these mice (96). In vitro studies have shown that Epo can directly affect neural progenitors, exerting a variety of effects that range from activating the basic helix-loop-helix transcription factor Mash1, which has been shown to activate quiescent NSCs (97), to increasing the nuclear translocation of nuclear factor- $\kappa \mathrm{B}$, which has been shown to promote neuronal differentiation (98), to increasing the proliferation and maturation of oligodendrocyte progenitors (99).

Epo is well known to be induced by hypoxia. For example, inducing systemic hypoxia in mice $\left(7 \% \mathrm{O}_{2}\right.$ for $\left.30 \mathrm{~min}\right)$ markedly increases Epo mRNA within $4 \mathrm{~h}$ after exposure that remains sustained for $>24 \mathrm{~h}$ (ref. 100). Similarly, exposing NSCs to hypoxia in vitro induces Epo expression concomitant with neuronal differentiation (98). Infusing Epo into the adult mouse lateral ventricle increased the number of progenitors, with a concomitant decrease in the number of NSCs, consistent with the view that Epo enhances NP maturation (98).

There are limited data available on the requirement for Epo in SVZ responses to pediatric models of brain injury; however, Epo is clearly required for the regenerative response of adult SVZ cells in a mouse model of stroke (96). There are several studies that show that exogenously administered Epo will enhance neurogenesis and oligodendrogenesis. For example, Iwai et al. (101) injected Epo (1,000 U/kg) intraperitoneally at multiple time points beginning $48 \mathrm{~h}$ after $\mathrm{H}-\mathrm{I}$ in postnatal day 7 rats. This delayed administration of Epo did not protect the brain from injury, but increased the production of $\mathrm{Dcx}+$ neuroblasts, oligodendrocyte progenitors, and enhanced the maturation of these oligodendrocyte progenitors (101). In a similar study, Kako et al. (99) performed retroviral-mediated fate-mapping studies in a P5 mouse model of $\mathrm{H}-\mathrm{I}$ and showed that intraperitoneal administration of asialo-Epo enhanced the maturation of SVZ-derived oligodendrocytes (99). As reviewed above, Gonzalez et al. (79) administered Epo in pediatric stroke model and found that Epo treatment significantly increased the production of neurons and oligodendrocytes from the NEPs while reducing the number of astrocytes produced (79).

\section{Interleukin-6}

Interleukin-6 (IL-6) exerts both proinflammatory and antiinflammatory actions and is secreted by $\mathrm{T}$ cells and macrophages to stimulate an immune response. The concentration of IL-6 in cerebrospinal fluid increases after birth asphyxia and H-I encephalopathy in human $(102,103)$. Maternal IL-6 can enter the fetal circulation, cross the blood-brain barrier, and affect central nervous system development $(104,105)$. For example, Gallagher et al. (106) administered a single pulse of IL- 6 by intraperitoneal injection into pregnant mice on gestational day 13.5, resulting in twice as many BrdU+ cells in the SVZ compared with controls at 2 months of age. IL-6 administration not only affects the SVZ but it also affects hippocampal neurogenesis (106).

Covey et al. (107) produced neonatal H-I injury in P6 rats, and investigated the role of neuroinflammation and more specifically IL-6 in the amplification of SVZ and subgranular zone progenitors with or without indomethacin administration, which is a cyclooxygenase 1 and 2 inhibitor. In that study, indomethacin reduced the accumulation of microglia/ macrophages and IL-6 production after H-I. Coincident with H-I, medially situated SVZ cells expanded in vehicle-treated animals. Indomethacin significantly decreased both the initial reactive increase in these progenitors and their ability to selfrenew. By contrast, indomethacin increased proliferation in the subgranular zone and lateral SVZ. These data showed that neuroinflammation and IL- 6 can have a positive effect on 


\section{Review Niimi and Levison}

primitive neural progenitor cell expansion after neonatal brain injury (107).

\section{Leukemia Inhibitory Factor}

Leukemia inhibitory factor (LIF) is a member of the IL-6 family of cytokines. Studies profiling the cytokine induction after perinatal brain injuries have shown that LIF is significantly increased within 1 day after neonatal and pediatric brain injury and thus precedes the increase in NSCs and progenitors (108). Astrocytes are activated by central nervous system injury and a single-molecule fluorescence in situ hybridization study for LIF and for GFAP showed that astrocytes are the major LIF expressing cells after neonatal $\mathrm{H}-$ I (51). The LIF that is secreted by the astrocytes may be essential for NP expansion after neonatal H-I (25). Although it is not clear whether IL-6 is expanding the abundance of NSCs, it is clear that LIF can expand the NP population (25). Loss-of-function studies using LIF heterozygous mice in the neonatal H-I model revealed that the expansion of MP3/ GRP2s, GRP3s, and MP2s that normally occurs after neonatal $\mathrm{H}-\mathrm{I}$ was blunted, indicating that these progenitors require LIF signaling to expand after injury (52).

\section{Transforming Growth Factor- $\alpha$}

TGF- $\alpha$ is an important cytokine that induces the proliferation of progenitors in the developing brain. In TGF- $\alpha$-null mouse, there are reduced numbers of proliferating SVZ cells, especially in the dorsomedial corner of the forebrain lateral ventricle, and fewer neuroblasts in the rostral migratory stream (109). TGF- $\alpha$ stimulates the EGF receptor, which is induced in models of $\mathrm{H}-\mathrm{I}$ injury and is necessary for NSC activation $(53,110,111)$.

Guerra-Crespo et al. (112) investigated the role of TGF- $\alpha$ in NSP responses to stroke (112) by infusing TGF- $\alpha 4$ weeks after injury. In this model, TGF- $\alpha$ produced a massive proliferative response, even when the growth factor was administered as late as 4 weeks after injury. They also showed greater BrdU incorporation in the corpus callosum and along the lateral wall of the ipsilateral ventricle compared with controls. Many of the BrdU-labeled cells were Nestin + with bipolar, elongated morphologies typical of migrating cells. At later time points of recovery, cells double labeled with both BrdU and NeuN could be observed in the injured striatum and many of these BrdU-labeled cells were also positive for Meis2 and DARPP-32, proteins known to be expressed in mature striatal neurons. TGF- $\alpha$ administered rats showed significant improvement in behavioral tests. These results showed that TGF- $\alpha$ induced a proliferative response in SVZ after stroke (112).

\section{Transforming Growth Factor- $\beta 1$}

Transforming growth factor- $\beta 1$ (TGF- $\beta 1$ ) is a pleiotropic cytokine that is produced by the choroid plexus, meninges, astrocytes, and microglia (113). Wrana et al. (114) evaluated the expression of the TGF- $\beta$ receptors on NSPs from the adult nervous system (114). They found that TGF- $\beta$ R1, R2, and R3 mRNAs were expressed by NSPs. When TGF- $\beta 1$ was added to neurosphere cultures, it diminished the growth rate to $25 \%$ in a dose-dependent manner. The growth inhibition was a result of reduced proliferation. However, sphere number was not affected by TGF- $\beta 1$, suggesting that the number of NSCs was not affected. Their data indicate that TGF- $\beta 1$ reduces cell proliferation with protracted growth arrest in NSP cultures.

Wachs et al. (115) infused TGF- $\beta 1$ into the lateral ventricles of adult rats for 7 days using osmotic pumps. During the infusion period, animals received BrdU injections. After 1 week of TGF- $\beta 1$ infusion, there was a marked reduction in the number of PCNA+ cells in the SVZ and fewer SVZ cells expressed Dcx. These effects of TGF- $\beta 1$ lasted at least for 4 weeks. Their in vitro studies further showed that SVZ progenitor cell proliferation was inhibited by TGF- $\beta 1$ without changing the self-renewal capacity of the NSCs or changing their fate (115).

Using the Vannucci model of perinatal H-I brain injury, where the common carotid artery is unilaterally cauterized followed by systemic hypoxia, Bain et al. (65) identified TGF$\beta 1$ as a cytokine that is produced during the subacute period of recovery from neonatal $\mathrm{H}-\mathrm{I}$, with levels of TGF- $\beta 1$ mRNA levels peaking between 2 and 7 days of recovery. In vitro studies by Bain et al. (65) showed that TGF- $\beta 1$ activates the type 1 activin-like kinase receptor-5 to stimulate the production of astrocytes from SVZ glial progenitors (65). Furthermore, increased levels of TGF- $\beta 1$ in the CSF subsequent to posthemorrhagic hydrocephalus correlate with increased pathology in premature newborns $(116,117)$ as well as with poor outcome from endoscopic third ventriculostomy (118). While TGF- $\beta$ is often associated with the M2-like microglial reaction, and thus, regarded as an anti-inflammatory cytokine, these data as well as other data on TGF- $\beta$ signaling in models of blood-brain barrier breakdown indicate that in the central nervous system TGF- $\beta$ may prevent or divert the normal generation of cells in the developing brain (119).

\section{Vascular Endothelial Cell Growth Factor- $\mathrm{A}$ and $-\mathrm{C}$}

Vascular endothelial cell growth factor-A (VEGF-A) production is regulated by a variety of stimuli such as hypoxia, growth factors, transformation, p53 mutation, estrogen, tumor promoters, and nitric oxide (120). While the VEGFs are generally associated with endothelial cell proliferation and angiogenesis, they also have been shown to have direct effects on the NPs of both the SVZ and the subgranular zone. For example, Bain et al. (121) reported that during the acute recovery period from neonatal $\mathrm{H}-\mathrm{I}$, both VEGF-A and VEGF-C were transiently induced in the SVZ. VEGF-A promoted the production of astrocytes from SVZ glial progenitors, while VEGF-C stimulated the proliferation of both early and late oligodendrocyte progenitors (121).

Both VEGF-A and VEGFR2 have been shown to be upregulated in the hippocampus after TBI and hypoxia $(122,123)$. Previous studies showed that TBI-induced hypoxia stimulates the production and release of VEGF-A (120). This TBI induced increase in VEGF-A may in turn also be responsible for the initial increase in neurogenesis within the 


\section{SVZ cell responses to brain injuries | ReView}

first few days after TBI. Lu et al. (123) showed that administering a VEGFR2 antagonist to block VEGF-A immediately after TBI returned the number of newborn neurons to control levels (123). These studies indicated the release of VEGF-A directly influences the production of newborn neurons after TBI.

\section{PROSPECTS FOR REPAIR}

For neonatal HIE, pediatric stroke and more severe TBIs, it will be essential to replace a wide variety of different neural cell types to restore neurological functional. To regenerate the complex cytoarchitecture of the neocortex, it will be necessary to produce new projection neurons in addition to new interneurons and oligodendrocytes. Based on data collected to date, it appears that the majority of the progenitors that migrate from the SVZ postnatally in response to most injuries either become interneurons or glial cells (124) or they do not survive long term $(47,48,55,56)$. The limited extent of cell replacement observed to date and the failure to observe the production of new projection neurons is a reflection of the maturational state of the postnatal brain. Developmental studies have established that the deep projection neurons are derived from the radial NEPs of the primitive ventricular zone and that as development proceeds the radial NEP cells become more restricted in their developmental potentials, such that as time progresses, they lose their ability to make early born neurons. Furthermore, it is becoming clearer that the residents of the inner SVZ make different subtypes of neurons than the residents of the outer SVZ. Thus, while some radial NEPs are present in the pediatric brain, they are not the same as the radial NEPs that were present when the brain first started to form. As discussed earlier, SVZ cells during the neonatal period give rise to granule layer and periglomerular layer interneurons that migrate to the olfactory bulb $(17,125)$. Of the newborn interneurons produced postnatally in the olfactory bulb, the vast majority are calretininpositive interneurons (126). Thus, the fact that the new neurons produced from the SVZ after injury are calretinin-positive interneurons should not be surprising, as this is entirely consistent with studies on normal neural development $(55,56)$.

A major limitation to cell replacement in the central nervous system is the requirement for cells produced in the germinal matrices that line the ventricles to migrate to more superficial regions of the brain. While such migration may occur in some late developing regions of the brain such as in the prefrontal neocortex, this pattern of migration is not widely observed. Another important consideration is that the radial NEP cells are not only stem cells and primitive progenitors, but during neural development they also provide an essential physical scaffold that a large fraction of immature neurons (especially pyramidal neurons) require to migrate from their periventricular origins into the neocortex and to subcortical structures. In the late fetal period in the human brain, and in the first postnatal week in the rodent brain, the radial glial scaffold collapses commensurate with a wave of astrocyte differentiation (127). A plausible explanation for the widespread migration of newly produced neurons in rodent models of perinatal $\mathrm{H}-\mathrm{I}$ is that the radial NEP processes have not yet retracted. Moreover, the fact that migration continues for months suggests that signals generated in the damaged brain are preventing (at least a subset of) the radial NEPs from retracting their processes. Consistent with the view that migration is occurring via radial NEP cells, Plane et al. (48) observed Dcx+ cells adjacent to GFAP+ astrocytes and suggested that they were using these glial cells to support their migration (48). Fagel et al. (87) also reported a similar phenomenon in immature rats recovering from chronic hypoxia, where the migrating cells were closely associated with GFAP+ cells (87). Ganat et al. (128) had earlier shown that there was an increase in cells expressing markers associated with radial glia after chronic hypoxia, and given the role of the radial cells in both neurogenesis and migration, they suggested that these cells were participating in the regeneration of neurons lost during the chronic hypoxia (128). Altogether, these observations suggest that replacing pyramidal neurons that have been lost to injury may be possible, but the radial NEPs will likely need to be either reprogrammed to a more primitive state or primitive radial cells will need to be transplanted into the SVZ. There are numerous labs working on these two strategies, but at this time, it is premature to predict which approach will be most fruitful.

Whereas there are significant barriers to replacing pyramidal neurons, replacing interneurons from the resident progenitors of the pediatric SVZ should not be difficult. As discussed above, there is significant tangential migration of neurons within six migratory streams in the neonatal brain. These tangential migrations do not require radial fibers. In gyrencephalic species, there are significant numbers of new neurons that are traveling to their final destinations. For example, in the postnatal day 14 piglet there are two million neuroblasts migrating through the subcortical white matter (12). These neuroblasts are adjacent to and migrate along blood vessels, and therefore, are not confined to radial migration (129). Similarly, there is substantial gliogenesis in the immature brain. What is clear from studies on the regenerative responses of the cells of the SVZ to pediatric injuries is that those responses are rapid and robust and the magnitude of this response is greater in the immature brain than in the mature brain. Therefore, while more studies are clearly needed, there is a strong likelihood that these studies can be translated into new therapeutics to improve the lives of children who have sustained a brain injury.

\section{ACKNOWLEDGMENTS}

This work was supported by funds provided by a grant from the New Jersey Commission on Brain Injury Research No. CBIR17IRG019.

Disclosure: The authors declare no conflict of interest.

\section{REFERENCES}

1. Beckung E, Carlsson G, Carlsdotter S, Uvebrant P. The natural history of gross motor development in children with cerebral palsy aged 1 to 15 years. Dev Med Child Neurol 2007;49:751-6. 
2. Takahashi T, Nowakowski RS, Caviness VS Jr. The cell cycle of the pseudostratified ventricular epithelium of the embryonic murine cerebral wall. J Neurosci 1995;15:6046-57.

3. Thomaidou D, Mione MC, Cavanagh JF, Parnavelas JG. Apoptosis and its relation to the cell cycle in the developing cerebral cortex. J Neurosci 1997; 17:1075-85.

4. Bayer SA, Altman J. Neocortical Development. New York: Raven Press, 1991.

5. Lewis PD, Lai M. Cell generation in the subependymal layer of the rat brain during the early postnatal period. Brain Res 1974;77:520-5.

6. Kershman J. The medulloblast and the medulloblastoma. Arch Neurol Psychiatry 1938;40:937-67.

7. Globus JH, Kuhlenbeck H. Subependymal cell plate (matrix) and its relation to brain tumors of ependymal type. J Neuropathol 1944;3:1-35.

8. Reid CB, Liang I, Walsh CA. Clonal mixing, clonal restriction, and specification of cell types in the developing rat olfactory bulb. J Comp Neurol 1999;403:106-18.

9. Altman J, Das GD. Autoradiographic and histological studies of postnatal neurogenesis: a longitudinal investigation of the kinetics, migration and transformation of cells incorporating thymidine in neonate rats, with special reference to postnatal neurogenesis in some brain regions. J Comp Neurol 1966;126:337-90.

10. Sanai N, Nguyen T, Ihrie RA, et al. Corridors of migrating neurons in the human brain and their decline during infancy. Nature 2011;478: 382-6.

11. De Marchis S, Fasolo A, Puche AC. Subventricular zone-derived neuronal progenitors migrate into the subcortical forebrain of postnatal mice. J Comp Neurol 2004;476:290-300.

12. Taylor SR, Smith C, Harris BT, Costine BA, Duhaime AC. Maturationdependent response of neurogenesis after traumatic brain injury in children. J Neurosurg Pediatr 2013;12:545-54.

13. Inta D, Alfonso J, von Engelhardt J, et al. Neurogenesis and widespread forebrain migration of distinct GABAergic neurons from the postnatal subventricular zone. Proc Natl Acad Sci USA 2008;105:20994-9.

14. Azim K, Fischer B, Hurtado-Chong A, et al. Persistent Wnt/beta-catenin signaling determines dorsalization of the postnatal subventricular zone and neural stem cell specification into oligodendrocytes and glutamatergic neurons. Stem Cells 2014;32:1301-12.

15. Snapyan M, Lemasson M, Brill MS, et al. Vasculature guides migrating neuronal precursors in the adult mammalian forebrain via brain-derived neurotrophic factor signaling. J Neurosci 2009;29:4172-88.

16. Whitman MC, Greer CA. Adult neurogenesis and the olfactory system. Progr Neurobiol 2009;89:162-75.

17. Merkle FT, Mirzadeh Z, Alvarez-Buylla A. Mosaic organization of neural stem cells in the adult brain. Science 2007;317:381-4.

18. Azim K, Hurtado-Chong A, Fischer B, et al. Transcriptional hallmarks of heterogeneous neural stem cell niches of the subventricular zone. Stem Cells 2015;33:2232-42.

19. Young GM, Levison SW. Persistence of multipotential progenitors in the juvenile rat subventricular zone. Dev Neurosci 1996;18:255-65.

20. Levison SW, Goldman JE. Multipotential and lineage restricted precursors coexist in the mammalian perinatal subventricular zone. J Neurosci Res 1997;48:83-94.

21. Zerlin M, Milosevic A, Goldman JE. Glial progenitors of the neonatal subventricular zone differentiate asynchronously, leading to spatial dispersion of glial clones and to the persistence of immature glia in the adult mammalian CNS. Dev Biol 2004;270:200-13.

22. Privat A, Leblond CP. The subependymal layer and neighboring region in the brain of the young rat. J Comp Neurol 1972;146:227-302.

23. Hubbard BM, Hopewell JW. Quantitative changes in the cellularity of the rat subependymal plate after X-irradiation. Cell Tissue Kinet 1980;13:403-13.

24. Doetsch F, Garcia-Verdugo JM, Alvarez-Buylla A. Cellular composition and three-dimensional organization of the subventricular germinal zone in the adult mammalian brain. J Neurosci 1997;17:5046-61.
25. Buono KD, Vadlamuri D, Gan Q, Levison SW. Leukemia inhibitory factor is essential for subventricular zone neural stem cell and progenitor homeostasis as revealed by a novel flow cytometric analysis. Dev Neurosci 2012;34:449-62.

26. Buono KD, Goodus MT, Moore L, Ziegler AN, Levison SMultimarker flow cytometric characterization, isolation and differentiation of neural stem cells and progenitors of the mouse subventricular zoneIn:Prusac Jed. Neural Surface Antigens: From Basic Biology Towards Biomedical Applications. New York: Elsevier Press, 2015: 175-85.

27. Ponti G, Aimar P, Bonfanti L. Cellular composition and cytoarchitecture of the rabbit subventricular zone and its extensions in the forebrain. J Comp Neurol 2006;498:491-507.

28. Costine BA, Missios S, Taylor SR, et al. The subventricular zone in the immature piglet brain: anatomy and exodus of neuroblasts into white matter after traumatic brain injury. Dev Neurosci 2015;37:115-30.

29. Fietz SA, Kelava I, Vogt J, et al. OSVZ progenitors of human and ferret neocortex are epithelial-like and expand by integrin signaling. Nat Neurosci 2010;13:690-9.

30. Smart IH, Dehay C, Giroud P, Berland M, Kennedy H. Unique morphological features of the proliferative zones and postmitotic compartments of the neural epithelium giving rise to striate and extrastriate cortex in the monkey. Cereb Cortex 2002;12:37-53.

31. Zecevic N, Rakic S, Jakovcevski I, Filipovic RContributions of the neocortical SVZ to human brain developmentInLevison SWed. Mammalian Subventricular Zones. New York: Springer, 2005: 117-60.

32. Parolisi R, Cozzi B, Bonfanti L. Non-neurogenic SVZ-like niche in dolphins, mammals devoid of olfaction. Brain Struct Funct 2017;222:2625-39.

33. Nowakowski TJ, Pollen AA, Sandoval-Espinosa C, Kriegstein AR. Transformation of the radial glia scaffold demarcates two stages of human cerebral cortex development. Neuron 2016;91:1219-27.

34. Bergmann O, Liebl J, Bernard S, et al. The age of olfactory bulb neurons in humans. Neuron 2012;74:634-9.

35. Kirschenbaum B, Nedergaard M, Preuss A, Barami K, Fraser RA, Goldman SA. In vitro neuronal production and differentiation by precursor cells derived from the adult human forebrain. Cereb Cortex 1994;4:576-89.

36. Menn B, Garcia-Verdugo JM, Yaschine C, Gonzalez-Perez O, Rowitch $\mathrm{D}$, Alvarez-Buylla A. Origin of oligodendrocytes in the subventricular zone of the adult Brain. J Neurosci 2006;26:7907-18.

37. Ernst A, Alkass K, Bernard S, et al. Neurogenesis in the striatum of the adult human brain. Cell 2014;156:1072-83.

38. Hagberg H, Mallard C, Ferriero DM, et al. The role of inflammation in perinatal brain injury. Nat Rev Neurol 2015;11:192-208.

39. Galinsky R, Bennet L, Groenendaal F, et al. Magnesium is not consistently neuroprotective for perinatal hypoxia-ischemia in term-equivalent models in preclinical studies: a systematic review. Dev Neurosci 2014;36:73-82.

40. Fang AY, Gonzalez FF, Sheldon RA, Ferriero DM. Effects of combination therapy using hypothermia and erythropoietin in a rat model of neonatal hypoxia-ischemia. Pediatr Res 2013;73:12-7.

41. Shankaran S, Laptook AR, Tyson JE, et al. Evolution of encephalopathy during whole body hypothermia for neonatal hypoxic-ischemic encephalopathy. J Pediatr 2012;160:567-572 e563.

42. Azzopardi DV, Strohm B, Edwards AD, et al. Moderate hypothermia to treat perinatal asphyxial encephalopathy. N Engl J Med 2009;361: 1349-58.

43. Shankaran S, Pappas A, McDonald SA, et al. Childhood outcomes after hypothermia for neonatal encephalopathy. N Engl J Med 2012;366: 2085-92.

44. Levison SW, Rothstein RP, Romanko MJ, Snyder MJ, Meyers RL, Vannucci SJ. Hypoxia/ischemia depletes the rat perinatal subventricular zone of oligodendrocyte progenitors and neural stem cells. Dev Neurosci 2001;23:234-47.

45. Romanko MJ, Rothstein RP, Levison SW. Neural stem cells in the subventricular zone are resilient to hypoxia/ischemia whereas progenitors are vulnerable. J Cereb Blood Flow Metab 2004;24:814-25. 
46. Brazel CY, Rosti RT, Boyce S, Rothstein RP, Levison SW. Perinatal hypoxia/ischemia damages and depletes progenitors from the mouse subventricular zone. Dev Neurosci 2004;26:266-74.

47. Ong J, Plane JM, Parent JM, Silverstein FS. Hypoxic-ischemic injury stimulates subventricular zone proliferation and neurogenesis in the neonatal rat. Pediatr Res 2005;58:600-6.

48. Plane JM, Liu R, Wang TW, Silverstein FS, Parent JM. Neonatal hypoxic-ischemic injury increases forebrain subventricular zone neurogenesis in the mouse. Neurobiol Dis 2004;16:585-95.

49. Yang Z, Levison SW. Hypoxia/ischemia expands the regenerative capacity of progenitors in the perinatal subventricular zone. Neuroscience 2006;139:555-64.

50. Felling RJ, Snyder MJ, Romanko MJ, et al. Neural stem/progenitor cells participate in the regenerative response to perinatal hypoxia/ischemia. J Neurosci 2006;26:4359-69.

51. Felling RJ, Covey MV, Wolujewicz P, Batish M, Levison SW. Astrocyteproduced leukemia inhibitory factor expands the neural stem/progenitor pool following perinatal hypoxia-ischemia. J Neurosci Res 2016;94: $1531-45$.

52. Buono KD, Goodus MT, Guardia Clausi M, Jiang Y, Loporchio D, Levison SW. Mechanisms of mouse neural precursor expansion after neonatal hypoxia-ischemia. J Neurosci 2015;35:8855-65.

53. Alagappan D, Lazzarino DA, Felling RJ, Balan M, Kotenko SV, Levison SW. Brain injury expands the numbers of neural stem cells and progenitors in the SVZ by enhancing their responsiveness to EGF. ASN Neuro 2009;1:e00009.

54. Yang Z, Levison SW. Perinatal hypoxic/ischemic brain injury induces persistent production of striatal neurons from subventricular zone progenitors. Dev Neurosci 2007;29:331-40.

55. Yang Z, You Y, Levison SW. Neonatal hypoxic/ischemic brain injury induces production of calretinin-expressing interneurons in the striatum. J Comp Neurol 2008;511:19-33.

56. Yang Z, Covey MV, Bitel CL, Ni L, Jonakait GM, Levison SW. Sustained neocortical neurogenesis after neonatal hypoxic/ischemic injury. Ann Neurol 2007;61:199-208.

57. Zaidi AU, Bessert DA, Ong JE, et al. New oligodendrocytes are generated after neonatal hypoxic-ischemic brain injury in rodents. Glia 2004;46:380-90.

58. Burtrum D, Silverstein FS. Hypoxic-ischemic brain injury stimulates glial fibrillary acidic protein mRNA and protein expression in neonatal rats. Exp Neurol 1994;126:112-8.

59. Back SA, Tuohy TM, Chen $\mathrm{H}$, et al. Hyaluronan accumulates in demyelinated lesions and inhibits oligodendrocyte progenitor maturation. Nat Med 2005;11:966-72.

60. Pendleton JC, Shamblott MJ, Gary DS, et al. Chondroitin sulfate proteoglycans inhibit oligodendrocyte myelination through PTPsigma. Exp Neurol 2013;247:113-21.

61. Zhou YX, Flint NC, Murtie JC, Le TQ, Armstrong RC. Retroviral lineage analysis of fibroblast growth factor receptor signaling in FGF2 inhibition of oligodendrocyte progenitor differentiation. Glia 2006;54:578-90.

62. Shimizu T, Kagawa T, Wada T, Muroyama Y, Takada S, Ikenaka K. Wnt signaling controls the timing of oligodendrocyte development in the spinal cord. Dev Biol 2005;282:397-410.

63. Gadea A, Aguirre A, Haydar TF, Gallo V. Endothelin-1 regulates oligodendrocyte development. J Neurosci 2009;29:10047-62.

64. Zhou YX, Armstrong RC. Interaction of fibroblast growth factor 2 (FGF2) and notch signaling components in inhibition of oligodendrocyte progenitor (OP) differentiation. Neurosci Lett 2007;421: 27-32.

65. Bain JM, Ziegler A, Yang Z, Levison SW, Sen E. TGF $\beta 1$ stimulates the over-production of white matter astrocytes from precursors of the "brain marrow" in a rodent model of neonatal encephalopathy. PLoS ONE 2010;5:e9567.

66. Sabo JK, Heine V, Silbereis JC, Schirmer L, Levison SW, Rowitch DH. Olig1 is required for noggin-induced neonatal myelin repair. Ann Neurol 2017;81:560-71.
67. Keenan HT, Bratton SL. Epidemiology and outcomes of pediatric traumatic brain injury. Dev Neurosci 2006;28:256-63.

68. Schneier AJ, Shields BJ, Hostetler SG, Xiang H, Smith GA. Incidence of pediatric traumatic brain injury and associated hospital resource utilization in the United States. Pediatrics 2006;118:483-92.

69. Bittigau P, Sifringer M, Pohl D, et al. Apoptotic neurodegeneration following trauma is markedly enhanced in the immature brain. Ann Neurol 1999;45:724-35.

70. Riggio S, Wong M. Neurobehavioral sequelae of traumatic brain injury. Mt Sinai J Med 2009;76:163-72.

71. Szele FG, Chesselet MF. Cortical lesions induce an increase in cell number and PSA-NCAM expression in the subventricular zone of adult rats. J Comp Neurol 1996;368:439-454.

72. Goodus MT, Guzman AM, Calderon F, Jiang Y, Levison SW. Neural stem cells in the immature, but not the mature, subventricular zone respond robustly to traumatic brain injury. Dev Neurosci 2015;37:29-42.

73. Tanaka N, Sasahara M, Ohno M, Higashiyama S, Hayase Y, Shimada M. Heparin-binding epidermal growth factor-like growth factor mRNA expression in neonatal rat brain with hypoxic/ischemic injury. Brain Res 1999;827:130-8.

74. Covey MV, Jiang Y, Alli VV, Yang Z, Levison SW. Defining the critical period for neocortical neurogenesis after pediatric brain injury. Dev Neurosci 2010;32:488-98.

75. Nelson KB. Perinatal ischemic stroke. Stroke 2007;38:742-5.

76. van der Aa NE, Benders MJ, Groenendaal F, de Vries LS. Neonatal stroke: a review of the current evidence on epidemiology, pathogenesis, diagnostics and therapeutic options. Acta Paediatr 2014;103:356-64.

77. Roach ES, Golomb MR, Adams R, et al. Management of stroke in infants and children: a scientific statement from a Special Writing Group of the American Heart Association Stroke Council and the Council on Cardiovascular Disease in the Young. Stroke 2008;39:2644-91.

78. Spadafora R, Gonzalez FF, Derugin N, Wendland M, Ferriero D, McQuillen P. Altered fate of subventricular zone progenitor cells and reduced neurogenesis following neonatal stroke. Dev Neurosci 2010;32:101-13.

79. Gonzalez FF, Larpthaveesarp A, McQuillen P, et al. Erythropoietin increases neurogenesis and oligodendrogliosis of subventricular zone precursor cells after neonatal stroke. Stroke 2013;44:753-8.

80. Warnes CA, Williams RG, Bashore TM, et al. ACC/AHA 2008 Guidelines for the Management of Adults with Congenital Heart Disease: Executive Summary: a report of the American College of Cardiology/American Heart Association Task Force on Practice Guidelines (writing committee to develop guidelines for the management of adults with congenital heart disease). Circulation 2008;118:2395-451.

81. Donofrio MT, Duplessis AJ, Limperopoulos C. Impact of congenital heart disease on fetal brain development and injury. Curr Opin Pediatr 2011;23:502-11.

82. Morton PD, Ishibashi N, Jonas RA, Gallo V. Congenital cardiac anomalies and white matter injury. Trends Neurosci 2015;38:353-63.

83. Bellinger DC, Wypij D, Rivkin MJ, et al. Adolescents with $d$ transposition of the great arteries corrected with the arterial switch procedure: neuropsychological assessment and structural brain imaging. Circulation 2011;124:1361-9.

84. Gilboa SM, Salemi JL, Nembhard WN, Fixler DE, Correa A. Mortality resulting from congenital heart disease among children and adults in the United States, 1999 to 2006. Circulation 2010;122:2254-63.

85. Stewart WB, Ment LR, Schwartz M. Chronic postnatal hypoxia increases the numbers of cortical neurons. Brain Res 1997;760:17-21.

86. Dean JM, McClendon E, Hansen K, et al. Prenatal cerebral ischemia disrupts MRI-defined cortical microstructure through disturbances in neuronal arborization. Sci Transl Med 2013;5:168ra167.

87. Fagel DM, Ganat Y, Silbereis J, et al. Cortical neurogenesis enhanced by chronic perinatal hypoxia. Exp Neurol 2006;199:77-91.

88. Bi B, Salmaso N, Komitova M, et al. Cortical glial fibrillary acidic protein-positive cells generate neurons after perinatal hypoxic injury. J Neurosci 2011;31:9205-21. 
89. Jablonska B, Scafidi J, Aguirre A, et al. Oligodendrocyte regeneration after neonatal hypoxia requires FoxO1-mediated p27Kip1 expression. J Neurosci 2012;32:14775-93.

90. Jablonska B, Gierdalski M, Chew LJ, et al. Sirt1 regulates glial progenitor proliferation and regeneration in white matter after neonatal brain injury. Nat Commun 2016;7:13866.

91. Scafidi J, Hammond TR, Scafidi S, et al. Intranasal epidermal growth factor treatment rescues neonatal brain injury. Nature 2014;506:230-4.

92. Aguirre A, Gallo V. Reduced EGFR signaling in progenitor cells of the adult subventricular zone attenuates oligodendrogenesis after demyelination. Neuron Glia Biol 2007;3:209-20.

93. Morton PD, Korotcova L, Lewis BK, et al. Abnormal neurogenesis and cortical growth in congenital heart disease. Sci Transl Med 2017: 9.

94. Hitoshi S, Alexson T, Tropepe V, et al. Notch pathway molecules are essential for the maintenance, but not the generation, of mammalian neural stem cells. Genes Dev 2002;16:846-58.

95. Androutsellis-Theotokis A, Leker RR, Soldner F, et al. Notch signalling regulates stem cell numbers in vitro and in vivo. Nature 2006;442:823-6.

96. Tsai PT, Ohab JJ, Kertesz N, et al. A critical role of erythropoietin receptor in neurogenesis and post-stroke recovery. J Neurosci 2006;26:1269-74.

97. Wang L, Zhang ZG, Gregg SR, et al. The Sonic hedgehog pathway mediates carbamylated erythropoietin-enhanced proliferation and differentiation of adult neural progenitor cells. J Biol Chem 2007;282:32462-70.

98. Anderson DJ, Gage F, Shingo T. Erythropoietin regulates the in vitro and in vivo production of neuronal progenitors by mammalian forebrain neural stem cells. Annu Rev Cell Dev Biol 2001;17:387-403.

99. Kako E, Kaneko N, Aoyama M, et al. Subventricular zone-derived oligodendrogenesis in injured neonatal white matter in mice enhanced by a nonerythropoietic erythropoietin derivative. Stem Cells 2012;30:2234-47.

100. Chikuma M, Masuda S, Kobayashi T, Nagao M, Sasaki R. Tissue-specific regulation of erythropoietin production in the murine kidney, brain, and uterus. Am J Physiol Endocrinol Metab 2000;279:E1242-8.

101. Iwai M, Stetler RA, Xing J, et al. Enhanced oligodendrogenesis and recovery of neurological function by erythropoietin after neonatal hypoxic/ischemic brain injury. Stroke 2010;41:1032-7.

102. Savman K, Blennow M, Gustafson K, Tarkowski E, Hagberg H. Cytokine response in cerebrospinal fluid after birth asphyxia. Pediatr Res 1998;43: 746-51.

103. Silveira RC, Procianoy RS. Interleukin-6 and tumor necrosis factoralpha levels in plasma and cerebrospinal fluid of term newborn infants with hypoxic-ischemic encephalopathy. J Pediatr 2003;143:625-9.

104. Banks WA, Kastin AJ, Gutierrez EG. Penetration of interleukin-6 across the murine blood-brain barrier. Neurosci Lett 1994;179:53-6.

105. Dahlgren J, Samuelsson AM, Jansson T, Holmang A. Interleukin-6 in the maternal circulation reaches the rat fetus in mid-gestation. Pediatr Res 2006;60:147-51.

106. Gallagher D, Norman AA, Woodard CL, et al. Transient maternal IL-6 mediates long-lasting changes in neural stem cell pools by deregulating an endogenous self-renewal pathway. Cell Stem Cell 2013;13:564-76.

107. Covey MV, Loporchio D, Buono KD, Levison SW. Opposite effect of inflammation on subventricular zone versus hippocampal precursors in brain injury. Ann Neurol 2011;70:616-26.

108. Covey MV, Levison SW. Leukemia inhibitory factor participates in the expansion of neural stem/progenitors after perinatal hypoxia/ischemia. Neuroscience 2007;148:501-9.

109. Tropepe V, Craig CG, Morshead CM, van der Kooy D. Transforming growth factor-alpha null and senescent mice show decreased neural progenitor cell proliferation in the forebrain subependyma. J Neurosci 1997;17:7850-9.

110. Guerra-Crespo M, Gleason D, Sistos A, et al. Transforming growth factor-alpha induces neurogenesis and behavioral improvement in a chronic stroke model. Neuroscience 2009;160:470-83.
111. Alagappan D, Felling RJ, Levison SW. Perinatal hypoxia/ischemia enhances EGF responsiveness of SVZ neural stem/progenitors. J Neurochem 2005;94:22.

112. Alagappan D, Balan M, Jiang Y, Cohen RB, Kotenko SV, Levison SW. Egr-1 is a critical regulator of EGF-receptor-mediated expansion of subventricular zone neural stem cells and progenitors during recovery from hypoxia-hypoglycemia. ASN Neuro 2013;5:183-193.

113. Constam DB, Philipp J, Malipiero UV, ten Dijke P, Schachner M, Fontana A. Differential expression of transforming growth factor-beta 1, -beta 2, and -beta 3 by glioblastoma cells, astrocytes, and microglia. J Immunol 1992;148:1404-10.

114. Wrana JL, Attisano L, Wieser R, Ventura F, Massague J. Mechanism of activation of the TGF-beta receptor. Nature 1994;370:341-7.

115. Wachs FP, Winner B, Couillard-Despres S, et al. Transforming growth factor-betal is a negative modulator of adult neurogenesis. J Neuropathol Exp Neurol 2006;65:358-70.

116. Whitelaw A. Intraventricular haemorrhage and posthaemorrhagic hydrocephalus: pathogenesis, prevention and future interventions. Semin Neonatol 2001;6:135-46.

117. Whitelaw A, Christie S, Pople I. Transforming growth factor-beta1: a possible signal molecule for posthemorrhagic hydrocephalus? Pediatr Res 1999;46:576-80.

118. Lipina R, Reguli S, Novackova L, Podesvova H, Brichtova E. Relation between TGF-beta 1 levels in cerebrospinal fluid and ETV outcome in premature newborns with posthemorrhagic hydrocephalus. Childs Nerv Syst 2010;26:333-41.

119. Levy N, Milikovsky DZ, Baranauskas G, et al. Differential TGF-beta signaling in glial subsets underlies IL-6-mediated epileptogenesis in mice. J Immunol 2015;195:1713-22.

120. Takahashi H, Shibuya M. The vascular endothelial growth factor (VEGF)/VEGF receptor system and its role under physiological and pathological conditions. Clin Sci 2005;109:227-41.

121. Bain JM, Moore L, Ren Z, Simonishvili S, Levison SW. Vascular endothelial growth factors $\mathrm{A}$ and $\mathrm{C}$ are induced in the SVZ following neonatal hypoxia-ischemia and exert different effects on neonatal glial progenitors. Transl Stroke Res 2013;4:158-70.

122. Marti HJ, Bernaudin M, Bellail A, et al. Hypoxia-induced vascular endothelial growth factor expression precedes neovascularization after cerebral ischemia. Am J Pathol 2000;156:965-76.

123. Lu KT, Sun CL, Wo PY, et al. Hippocampal neurogenesis after traumatic brain injury is mediated by vascular endothelial growth factor receptor-2 and the Raf/MEK/ERK cascade. J Neurotrauma 2011;28:441-50.

124. Teramoto T, Qiu J, Plumier JC, Moskowitz MA. EGF amplifies the replacement of parvalbumin-expressing striatal interneurons after ischemia. J Clin Invest 2003;111:1125-32.

125. Kohwi M, Petryniak MA, Long JE, et al. A subpopulation of olfactory bulb GABAergic interneurons is derived from Emx1- and Dlx5/6expressing progenitors. J Neurosci 2007;27:6878-91.

126. Batista-Brito R, Close J, Machold R, Fishell G. The distinct temporal origins of olfactory bulb interneuron subtypes. J Neurosci 2008;28: 3966-75.

127. Tramontin AD, Garcia-Verdugo JM, Lim DA, Alvarez-Buylla A. Postnatal development of radial glia and the ventricular zone (VZ): a continuum of the neural stem cell compartment. Cereb Cortex 2003;13: 580-7.

128. Ganat Y, Soni S, Chacon M, Schwartz ML, Vaccarino FM. Chronic hypoxia up-regulates fibroblast growth factor ligands in the perinatal brain and induces fibroblast growth factor-responsive radial glial cells in the sub-ependymal zone. Neuroscience 2002;112:977-91.

129. Bovetti S, Hsieh YC, Bovolin P, Perroteau I, Kazunori T, Puche AC. Blood vessels form a scaffold for neuroblast migration in the adult olfactory bulb. J Neurosci 2007;27:5976-80. 